ISSN: 2643-6736

\title{
Editorial on Self-Healing Robots
}

\author{
Manu Mitra* \\ Department of Electrical Engineering, Alumnus of University of Bridgeport, Bridgeport, United States
}

*Corresponding author: Manu Mitra, Department of Electrical Engineering, Alumnus of University of Bridgeport, Bridgeport, United States

\section{Introduction}

Scientists at University of Colorado, Boulder had developed a first and fully re-healable and recyclable electronic material. This novel technology functions like properties of human skin, capable of measuring pressure, temperature and vibration. Technically, it's a self-healing Robot - For instance if robots are wrapped in an electronic material that mimics as human skin; theoretically it can also sense objects that is too hot or too cold or if more or less pressure needs to be exerted on an object. If this material is beyond repair, then it can be soaked in a solution that separates out the silver nanoparticles then be fully recycled into new usable material [1] (Figure 1).

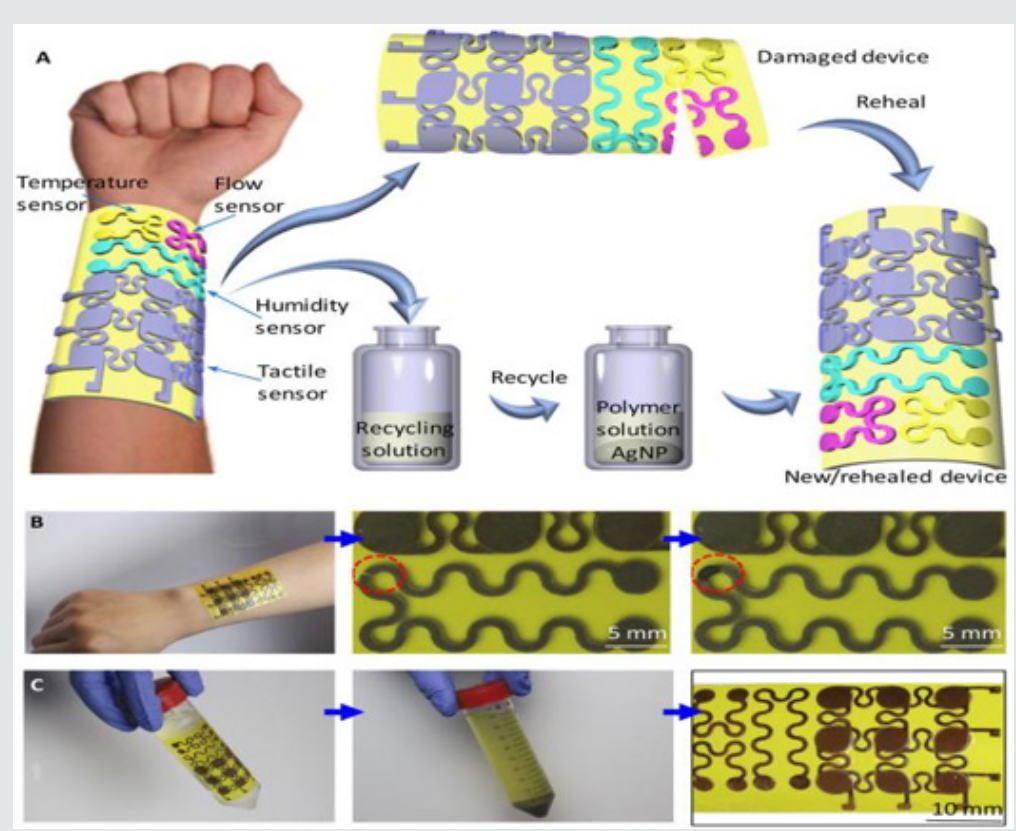

Figure 1: Illustrates self-healing material developed at University of Colorado, Boulder. Image Credit: Quartz [1].

\section{Self-Healing Robots Breakthrough}

Many of natural organisms have the capability to heal themselves. Now, manmade machines will be able to imitate this phenomenon. In the research Carnegie Mellon University created a self-healing material that spontaneously heals itself even under extreme mechanical damage. The soft composite material is consisting of liquid metal droplets suspended in a soft elastomer. When it is broken, the droplets rupture to form a new arrangement with adjacent droplets and redirect electrical signals without any disruption. Circuits which are created with conductive traces of such kind of material remain entirely and continuously functioning when punctured, severed or material removed. Applications for this kind of novel technology includes bio-inspired robotics, wearable, computing human and machine interaction. Because material also exhibits high electrical conductivity; it does not change when starched, theoretically, it is ideal for use in power and data transmission. 
"If we have to build machines that are more compatible with the human body and natural atmosphere, then we have to begin with new material types" was confirmed by the author of the study [2,3] (Figure 2).

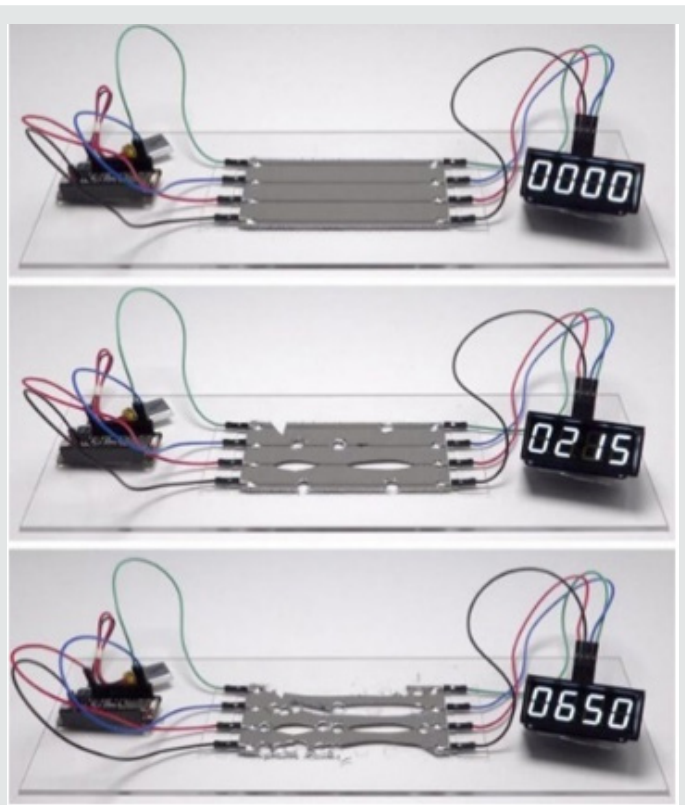

Figure 2: Illustrates a digital clock continues to run as damaged circuits instantaneously heal themselves, rerouting electrical signals without interruption. Image Credit: Nature Materials [3].

\section{Delicate, Soft and Self-Healing Robot}

A new class of delicate and soft, electrically activated robots are capable of imitating the expansion and contraction of natural muscles. These robots which can be designed from a wide range of low-cost materials and are able to self-sense and self-heal from electrical damage, representing a major advance in soft robotics. A challenge in the field of "soft robotics" that can imitate the versatility and performance. Nevertheless, Keplinger Research Group in the College of Engineering and Applied Science has created a novel method of a new class of soft robotics. This novel method of hydraulically intensified self-healing electrostatic actuators abjure bulky, rigid pistons and motors of conventional robots for soft structures that respond to applied voltage with comprehensive range of motions. These soft robots can accomplish a variety of jobs including holding subtle objects for instance, raspberry and a raw egg as well as lifting heavy objects. These new tech robots actuators exceed the strength, speed and efficiency of biological muscle and their flexibility. In addition to assisting as hydraulic fluid which supports flexible movements, the use of liquid insulating layer enables robots' actuators to self-heal from electrical damage. Another soft actuator that are controlled by high voltage, also known as dielectric elastomer actuators; it utilizes a solid insulating layer that fails catastrophically from electrical damage [4-6] (Figure 3).

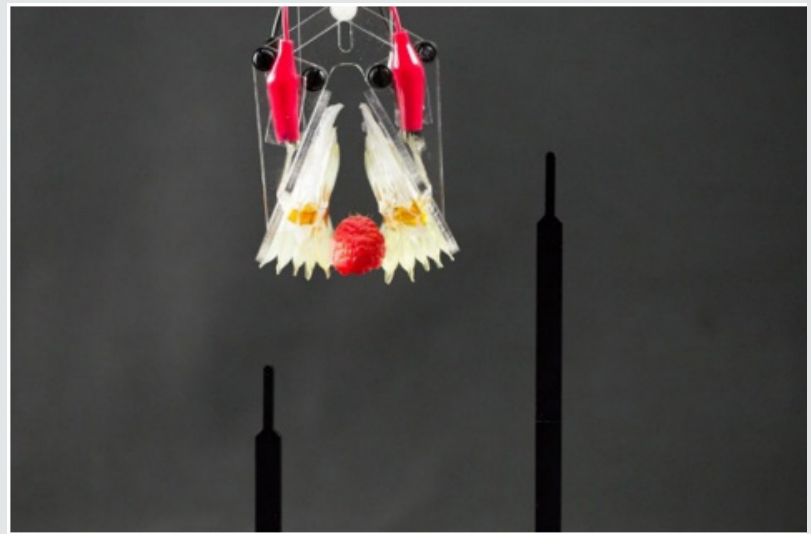

Figure 3: Illustrates actuators can be designed as soft grippers to handle and manipulate delicate objects, like this raspberry. Image Credit: Keplinger Lab / University of Colorado Boulder [6].

\section{Graphene for Artificial Skin in Self-Healing Robots}

Graphene is a sheet of pure carbon atoms and it is known as world's strongest material; it is one million times thinner than paper. It is so thin that it can be viewed as two dimensional. Although, it's hefty price, graphene has become most favorable nanomaterials due to its unique and multipurpose applications. One of the organs in the human body; skin is known for its fascinating self-healing properties. Imitating this phenomenon has proved too much of work as manmade materials lack this technology. Due to stretching or bending and incidental scratches, artificial skin used in robots are too much susceptible to ruptures and fissures. In this study a novel solution where a sub-nano sensor uses graphene to sense a crack as soon as it starts nucleation even after the crack has spread a certain distance. Scientist subjected a single layer graphene comprising various issues like pre-existing vacancies and inversely oriented pre-existing cracks to uniaxial tensile loading till fracture. Once it is completed, graphene started to heal and the self-healing continued irrespective of the nature of pre-existing issues in the graphene sheet. Not to mention, whatever the length of crack, they were all healed; provided the critical crack opening distance are within 0.3 to $0.5 \mathrm{~nm}$ for pristine sheet and sheet with pre-existing defects $[7,8]$.

\section{Wolverine Inspired Material for Self-Healing Robot}

Researchers at University of California, Riverside designed a novel method of an ionic conductor, that means material that ions can flow through and it is mechanically stretchable, transparent and self-healing. These materials have wide variety of applications in extensive range of fields. It can give robots to self-heal after mechanical failure. It also extends the lifetime of lithium ion batteries used in electronics and electric cars; and improve biosensors that are used in medical and environmental fields. This material was inspired by wound healing in nature, self-healing materials repair the damage caused by wear and extend the lifetime 
and in turn lowering the cost of the device. Ionic conductors are a class of materials with vital roles in energy storage, sensors, solar energy conversion and electronic devices.

The vital problem is the identification of bonds that are stable and reversible under electrochemical conditions. Traditionally, selfregenerative polymers utilize non-covalent bonds which makes it difficult because these bonds are affected by electrochemical reactions that decreases the performance of the materials. Wang solved that critical problem by utilizing a novel mechanism that is known as ion-dipole interactions; the forces between charged ions and polar molecules that are highly stable under electrochemical conditions. In this method, he joined a stretchable polymer with mobile and high ionic strength salt to create material with the properties that scientist was searching. It is a low cost and can be easily produce soft rubber like material that can stretch 50 times its original length. If it has been cut, it can completely heal in 24 hours at room temperature. As a matter of fact, after only five minutes of healing the material can be stretched two times its original length $[9,10]$ (Figure 4).
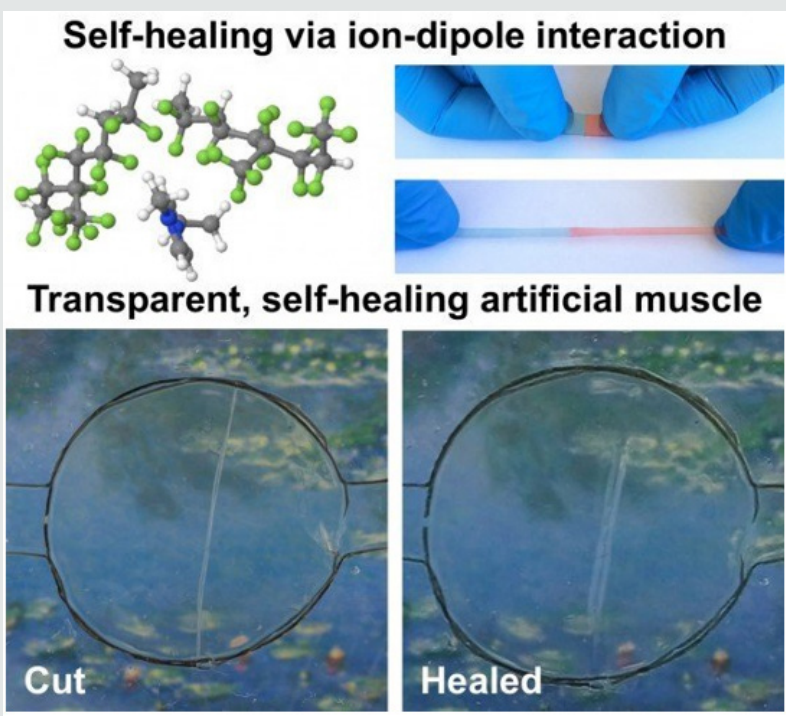

Figure 4: Illustrates showing self-healing via ion-dipole interaction. Image Credit: University of Colorado, Boulder [10].

\section{Robots That Can Morph Metal Shapes}

Researchers created a novel hybrid material that is stiff metal, soft and porous rubber foam that integrates the best properties of stiffness and as well as elasticity when a change of shape is necessary. This material also has the capability of self-heal if damaged. This material integrates with the soft alloy called Field's metal with a porous silicone foam and the rigidity and load bearing capability of humans with the capability to drastically alter shape, like an octopus. In addition to this, its melting point is 144 degrees Fahrenheit, the major reason Field's metal was taken because it has no lead in it. The elastomer foam is immersed into a molten metal then it is placed in a vacuum so that the air in the foam's pores are removed and interchanged by the alloy. The foam had pore sizes of around 2 millimeters that can be adjusted to make a stiffer or more flexible material. In testing of its strength and elasticity the material ability was deformed when heated above 144 degrees, then it regained rigidity when cooled; then it returned to its original shape and strength when reheated [11,12] (Figure 5).

\section{Acknowledgment}

Author would like to thank Prof. Navarun Gupta, Prof. Hassan Bajwa, Prof. Linfeng Zhang and Prof. Hmurcik for their academic support. Author also thanks anonymous reviewers for their comments.

\section{References}

1. Epstein A (2018) Self-healing electronic skin will help robots have a sense of touch like humans.

2. Eric J Markvicka, Michael D. Bartlett, Xiaonan Huang, Carmel Majidi (2018) An autonomously electrically self-healing liquid metal-elastomer composite for robust soft-matter robotics and electronics. Nature Materials 17: 618-624.

3. College of Engineering, Carnegie Mellon University (2018) Self-healing material a breakthrough for bio-inspired robotics. ScienceDaily.

4. E Acome, SK Mitchell, TG Morrissey, MB Emmett, C Benjamin, et al. Hydraulically amplified self-healing electrostatic actuators with musclelike performance. Science 359(6371): 61-65.

5. Nicholas Kellaris, Vidyacharan Gopaluni Venkata, Garrett M. Smith, Shane K (2018) Mitchell and Christoph Keplinger. Peano-HASEL actuators: Muscle-mimetic, electrohydraulic transducers that linearly contract on activation. Science Robotics 3(14): eaar3276.

6. University of Colorado at Boulder (2018) Soft, self-healing devices mimic biological muscles: A new class of soft, electrically activated devices mimics the expansion and contraction of natural muscles. ScienceDaily.

7. K Vijaya Sekhar, Swati Ghosh Acharyya, Sanghamitra Debroy, V Pavan Kumar Miriyala, Amit Acharyya (2016) Self-healing phenomena of graphene: potential and applications. Open Physics 14(1).

8. De Gruyter Open (2017) Self-healing graphene holds promise for artificial skin in future robots. ScienceDaily.

9. Yue Cao, Timothy G Morrissey, Eric Acome, Sarah I Allec, Bryan M Wong, et al. (2016) A Transparent, Self-Healing, Highly Stretchable Ionic Conductor. Advanced Materials 29(10).

10. University of California, Riverside (2016) A wolverine-inspired material: Self-healing, transparent, highly stretchable material can be electrically activated. ScienceDaily.

11. Ilse M Van Meerbeek, Benjamin C Mac Murray, Jae Woo Kim, Sanlin S Robinson, Perry X Zou, et al. (2016) Morphing Metal and Elastomer Bicontinuous Foams for Reversible Stiffness, Shape Memory, and SelfHealing Soft Machines. Advanced Materials 28(14): 2801-2806.

12. Cornell University (2016) Morphing metal shapes future of soft robotics. ScienceDaily. 
(C) (P) This work is licensed under Creative

To Submit Your Article Click Here: Submit Article

DOI: 10.32474/ARME.2019.01.000124

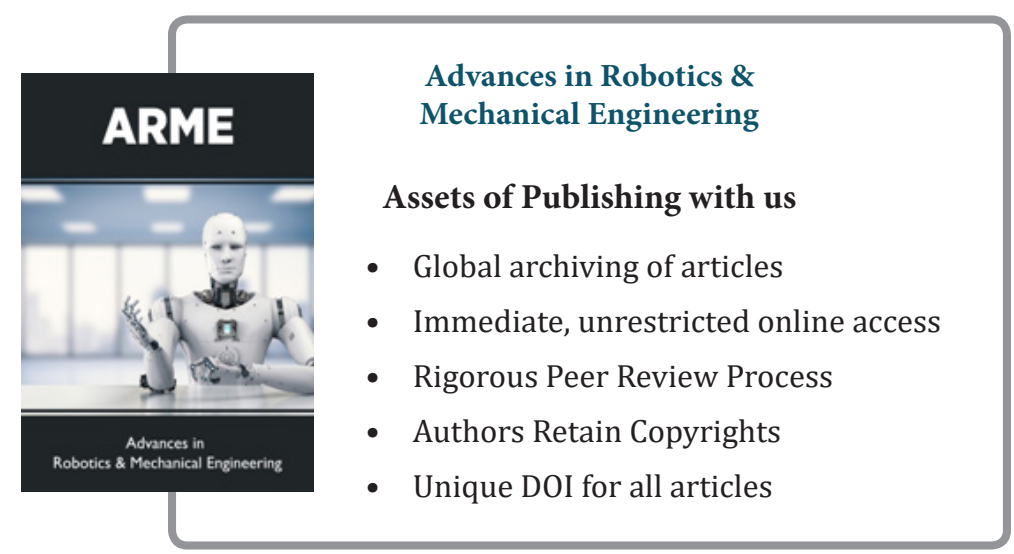

